ARTIGO ORIGINAL

Revista de Enfermagem do Centro-Oeste Mineiro

2021; $11 / 3484$

DOI: http://dx.doi.org/10.19175/recom.v11i0.3484

www.ufsj.edu.br/recom

\title{
Utilização de indicadores de qualidade: dificuldades e estratégias na voz de enfermeiros-líderes
}

\author{
The use of quality indicators: difficulties and strategies in the voice of nurse-leaders
}

\author{
Uso de indicadores de calidad: dificultades y estrategias en la voz de los enfermeros líderes
}

\section{RESUMO}

Objetivo: Conhecer as dificuldades e estratégias dos enfermeiros-líderes na utilização dos indicadores de qualidade em ambiente hospitalar. Método: Pesquisa qualitativa, exploratório-descritiva, realizada em hospital filantrópico do Sul do Brasil, na qual participaram 12 enfermeiros. Adotaram-se entrevistas semiestruturadas e grupo focal para a coleta de dados, os quais foram analisados conforme análise de conteúdo. Resultados: Os enfermeiros evidenciaram como dificuldades a subnotificação de eventos adversos, os limites na interpretação e aplicabilidade dos dados, falta de tempo e falta de compartilhamento das informações na utilização dos indicadores de qualidade. Já como estratégias compartilharam a construção e discussão coletiva, o envolvimento da equipe de enfermagem e saúde, o feedback entre os profissionais e a educação permanente. Conclusão: É necessária a sensibilização dos gestores das instituições de saúde, para a instrumentalização dos enfermeiros, de maneira a desenvolver competências necessárias para a melhor utilização dos indicadores de qualidade, bem como investimentos para superar as dificuldades.

Descritores: Indicadores de Qualidade em Assistência à Saúde; Qualidade da Assistência à Saúde; Gestão em Saúde; Enfermagem.

\section{ABSTRACT}

Objective: To know the difficulties and strategies nurse-leaders face using quality indicators in the hospital environment. Method: A qualitative, exploratory-descriptive research was conducted in a philanthropic hospital in southern Brazil. 12 nurses participated in this study. Semi-structured interviews and focus group for data collection were adopted and analyzed according to content analysis. Results: The nurses highlight as difficulties the underreporting of adverse events, the limits on data interpretation and applicability, the lack of time, and the lack of information sharing about the use of quality indicators. As strategies, they point out construction and collective discussions, the mutual involvement of the nursing and health team, feedback between professionals, and continuing education. Conclusion: It is necessary to raise awareness of managers of health institutions regarding the instrumentalization of nurses, so as to develop skills necessary for the best use of quality indicators, as well as investments to overcome difficulties.

Descriptors: Quality Indicators; Health Care; Quality of Health Care; Health Management; Nursing.

\section{RESUMEN}

Objetivo: Conocer las dificultades y estrategias de los enfermeros líderes para el uso de indicadores de calidad en el entorno hospitalario. Método: Investigación cualitativa, exploratoria-descriptiva realizada en un hospital filantrópico en el sur de Brasil, en el que participaron 12 enfermeros. Se adoptaron entrevistas semiestructuradas y grupos focales para la recopilación de datos, que se analizaron según el análisis de contenido. Resultados: Los enfermeros destacan como dificultades la falta de notificación de eventos adversos, los límites en la interpretación y aplicabilidad de los datos, la falta de tiempo y la falta de intercambio de información en el uso de indicadores de calidad. Así como estrategias que comparten la construcción y la discusión colectiva, la participación del equipo de enfermería y salud, la retroalimentación entre los profesionales y la educación continua. Conclusión: Es necesario sensibilizar a los gerentes de las instituciones de salud para la instrumentalización de los enfermeros, a fin de desarrollar las habilidades necesarias para el mejor uso de los indicadores de calidad, así como las inversiones para superar las dificultades presentadas.

Descriptores: Indicadores de Calidad de la Atención de Salud; Calidad de la Atención de Salud; Gestión en Salud; Enfermería.
Ana Cristina Pretto Báo ${ }^{1}$

0000-0002-2747-7197

Simone Coelho Amestoy ${ }^{2}$

O0000-0001-8310-2157

Letícia de Lima Trindade ${ }^{3}$

D) 0000-0002-7119-0230

Gisela Maria Schebella Souto de

Moura ${ }^{1}$

0000-0002-4744-478X

\section{Andrieli Daiane Zdanski de \\ Souza ${ }^{1}$ \\ 0000-0002-3235-0182}

1 Universidade Federal do Rio Grande do Sul.

2 Universidade Federal do Vale do São Francisco.

3 Universidade do Estado de Santa Catarina.

Autor correspondente: Ana Cristina Pretto Báo E-mail:anacbao@yahoo.com.br

\begin{abstract}
Como citar este artigo:
Báo ACP, Amestoy SC, Trindade LL, et al. Utilização de indicadores de qualidade: dificuldades e estratégias na voz de enfermeiros-líderes. 2021;11:e3484. Available in: [Access http://dx.doi.org/10.19175/recom.v11i $\underline{0.3484}$
\end{abstract}


2|Bá o ACP, Amestoy SC, Trindade LL, et al.

\section{INTRODUÇÃO}

As organizações de saúde estão cada vez mais comprometidas com a prestação de cuidados efetivos e a priorização da segurança do paciente. Esse fato acarretou o reconhecimento da importância dos sistemas de gestão da qualidade, que são vistos como um conjunto de atividades que servem para monitorar e controlar a qualidade dos cuidados $^{(1-3)}$. Com isso, os indicadores de qualidade constituem-se um suporte importante e relevante dentro dos serviços de saúde, atrelados à maioria dos processos que envolvem a assistência aos pacientes, com amplitude operacional e de gestão(4). Neste sentido, as instituições que buscam certificações precisam trabalhar os indicadores como componentes positivos da assistência, visto que a sua utilização demonstra uma cultura de segurança arraigada da organização(5).

O gestor de enfermagem, quando fundamenta sua atuação em avaliações periódicas, por meio dos indicadores, impulsiona os profissionais na busca por melhoria dos serviços, além de permitir a compreensão da qualidade assistencial(6). Assim, o enfermeirolíder, ao utilizar os indicadores de qualidade, durante o percurso do cuidado, pode transformar os resultados da assistência.

O enfermeiro-líder consiste no profissional capaz de influenciar de forma construtiva os demais integrantes da equipe de enfermagem e saúde, com 0 intuito de qualificar o gerenciamento do cuidado e de fortalecer as relações interpessoais no trabalho ${ }^{(7)}$. Com isso, potencializa os resultados melhores para a assistência, mediante a utilização de instrumentos gerenciais, tais como os indicadores de qualidade.

Nesta perspectiva, um estudo revela a importância da utilização de indicadores para avaliar a qualidade dos processos assistenciais. $\mathrm{Na}$ percepção dos enfermeiros, os indicadores são ferramentas capazes de melhorar as ações assistenciais da equipe de enfermagem, demonstrando a conscientização do profissional quanto às exigências do mercado e às necessidades de qualificação dos serviços de saúde ${ }^{(8)}$

Pesquisas revelam a importância singular dos indicadores de qualidade para a assistência de enfermagem em diferentes cenários assistenciais $^{(3-4,9)}$. Ainda, o sistema de gestão da qualidade é apontado como importante para a melhoria da qualidade clínica, em hospitais da Europa, assim como promovem o trabalho em equipe e o clima de segurança institucional ${ }^{(1)}$.

Neste sentido, o Ministério da Saúde brasileiro, em 2013, lançou a Política de Atenção Hospitalar (PNHOSP), com vista a fortalecer a avaliação dos serviços, sendo a utilização dos indicadores de qualidade, no âmbito hospitalar, uma alternativa para a qualificação das instituições. A PNHOSP estabelece as diretrizes para a organização do componente hospitalar na Rede de Atenção à Saúde e defende que a assistência hospitalar, no Sistema Único de Saúde (SUS), deverá ser organizada, a partir das necessidades da população, com a finalidade de garantir $\mathrm{o}$ atendimento aos usuários ${ }^{(10)}$.

A gestão hospitalar deve ser pautada na garantia ao acesso e à qualidade da assistência, ao cumprimento de metas pactuadas, à eficiência e transparência da aplicação dos recursos e ao planejamento participativo e democrático ${ }^{(10)}$. Diante do que foi exposto, cabe aos hospitais desenvolver estratégias para o monitoramento e a avaliação dos compromissos e das metas pactuadas e da qualidade das ações e dos serviços de forma sistemática. No entanto os gestores de saúde, em especial, os enfermeiros-líderes, muitas vezes, não estão preparados ou deparam-se com muitas dificuldades para trabalhar com a avaliação da qualidade, por meio dos indicadores de qualidade.

Sinaliza-se que existem alguns estudos que investigam a utilização dos indicadores de qualidade no ambiente hospitalar e suas dificuldades. Pesquisas ${ }^{(8,11-12)}$ realizadas com enfermeiros registram que a utilização dos indicadores de qualidade já é prática nas instituições de saúde, porém ainda é necessária a implementação de estratégias de análise dos indicadores, para que sejam passíveis de comparabilidade e que são encontradas dificuldades na sua utilização. Assim, entende-se que é necessário o empoderamento dos enfermeiros-líderes acerca dos indicadores de qualidade, para que possam propor melhorias ao gerenciamento do cuidado em saúde, no que tange ao atendimento das necessidades dos usuários do sistema hospitalar.

O compartilhamento das informações obtidas pelos indicadores e a discussão com a equipe de enfermagem pode ser adotada como estratégia, para identificar fragilidades e estabelecer metas, visando à melhoria da assistência prestada ${ }^{(6)}$. Observa-se que o controle 
da qualidade, realizado por meio da análise de indicadores, representa uma estratégia fundamental na busca de uma assistência segura, da objetividade comprovada por evidências e do gerenciamento dos resultados ${ }^{(13)}$.

O presente artigo justifica-se, a fim de buscar suprir as lacunas encontradas nos estudos científicos $^{(8,11-13)}$ e comparar o tema indicadores de qualidade com a PNHOSP. Além disso, almejase que o estudo possa contribuir com os enfermeiros-líderes das instituições hospitalares, na utilização dos indicadores de qualidade assistencial, como uma ferramenta para fundamentar a tomada de decisão, o planejamento e a organização dos serviços. Portanto este estudo teve como objetivos avaliar quais as dificuldades e estratégias dos enfermeiros-líderes, na utilização dos indicadores de qualidade, para o gerenciamento do cuidado, no âmbito hospitalar, além de conhecer as dificuldades e estratégias dos enfermeiros-líderes para a utilização dos indicadores de qualidade no ambiente hospitalar.

\section{MÉTODO}

Trata-se de uma pesquisa qualitativa do tipo exploratório-descritiva, realizada em um hospital filantrópico da Serra gaúcha/Rio Grande do Sul, de caráter geral e referência para urgência, emergência e alta complexidade, com 293 leitos. A escolha por esse local deu-se por ser uma instituição de ensino, creditada nível 2 pela Organização Nacional de Acreditação (ONA) e que tem mobilizado ações voltadas para a qualidade, gestão de riscos e segurança do paciente. Além disso, acompanha e monitora indicadores de qualidade, como taxa de infecção hospitalar, média de permanência hospitalar, satisfação do cliente, incidência de quedas, incidência de flebite, entre outros.

A instituição conta com sete unidades de internação, totalizando 29 enfermeiros-líderes. Para a seleção dos participantes, foram adotados os seguintes critérios: ser enfermeiro-líder, estar atuando por, no mínimo, um ano na instituição e trabalhar nas unidades de internação. Não foram incluídos, no estudo, enfermeiros que estavam afastados por férias, licença-maternidade, licençasaúde, no momento da coleta de dados. Assim, compuseram a população do estudo 12 enfermeiras.

Os dados foram coletados por meio de entrevista semiestruturada e grupo focal no período de março a maio de 2016. A entrevista seguiu o roteiro de questões: "Como são utilizados os indicadores de qualidade?", "Existem dificuldades na utilização de indicadores?" e "Quais são as estratégias para a utilização dos indicadores de qualidade que contribuem ao gerenciamento de boas práticas em saúde?". Primeiramente foram feitas as entrevistas com todas as participantes e, na sequência, foram convidadas a participar do grupo focal. Nessa etapa, sete enfermeiras participaram de duas sessões do grupo focal, as quais tiveram como objetivo apresentar os dados emergidos das entrevistas, a fim de realizar validação e verificar o surgimento de novas percepções acerca do tema.

A coleta de dados das duas etapas ocorreu em local reservado da instituição, e as discussões foram gravadas em áudio e transcritas na íntegra. Os depoimentos advindos das entrevistas duraram, em média, 30 minutos, e as sessões dos grupos focais uma hora. As entrevistas e o grupo focal foram conduzidos pelas pesquisadoras. Os depoimentos oriundos das entrevistas foram identificados pela letra $E$ (entrevista) e o número ordinal da sua realização (E1, E2..) e os achados do grupo focal foram identificados com as letras GF mais o número ordinal representando cada enfermeiro (GF1, GF2...), preservando assim o anonimato. No final de cada sessão do GF, a coordenadora e uma pesquisadora observadora reuniam-se, com o intuito de realizar um feedback sobre o que transcorreu no grupo.

Para a análise dos dados, realizou-se uma leitura minuciosa das entrevistas semiestruturadas e dos depoimentos do grupo focal, conforme análise de conteúdo ${ }^{(14)}$. Inicialmente, foi realizada a fase exploratória de investigação e, após, o momento interpretativo, do qual utilizaram-se as falas dos participantes. Depois disso, realizou-se a ordenação dos dados que englobaram a transcrição do material das entrevistas semiestruturadas e do grupo focal, pela leitura horizontal e exaustiva dos textos. $\mathrm{Na}$ leitura transversal, os dados foram separados por unidades de sentido. Logo após o processo classificatório, foram identificadas as categorias, juntando as partes semelhantes, buscando-se as conexões e guardando-as em códigos. Compuseram esse manuscrito as seguintes categorias: Dificuldades evidenciadas pelas enfermeiras-líderes na utilização dos indicadores de qualidade e Estratégias para a utilização dos indicadores de qualidade. 
Na discussão, os dados foram interpretados à luz da literatura e com a PNHOSP.

A pesquisa foi aprovada pelo Comitê de Ética em Pesquisa, sob o número de CAAE 51678715.4.3001.5331 e, para o seu desenvolvimento, foram atendidos os preceitos éticos instituídos na Resolução 466/12 do Conselho Nacional de Saúde, com a utilização do termo de consentimento livre e esclarecido.

\section{RESULTADOS E DISCUSSÃO}

Os participantes do estudo, em sua totalidade, eram do sexo feminino e a idade variou entre 26 e 50 anos, com média de 32,5 anos. O tempo de formação oscilou de dois a nove anos, com média de 5,6 anos. Em relação ao tempo em que possui vínculo empregatício com o hospital, os participantes tinham, em média, 4,6 anos de atuação no cenário (1-11 anos).

Na sequência, apresentam-se as categorias e subcategorias originadas do processo de análise dos dados coletados.

Quadro 1 - Categorias e subcategorias elencadas da pesquisa

\begin{tabular}{|c|c|}
\hline Categorias & Subcategorias \\
\hline \multirow{4}{*}{$\begin{array}{l}\text { Dificuldades na } \\
\text { utilização dos } \\
\text { indicadores de } \\
\text { qualidade }\end{array}$} & Subnotificação \\
\hline & $\begin{array}{l}\text { Interpretação e aplicabilidade } \\
\text { dos indicadores }\end{array}$ \\
\hline & Falta de tempo \\
\hline & $\begin{array}{l}\text { Falta de compartilhamento dos } \\
\text { dados entre a equipe de } \\
\text { enfermagem e multidisciplinar } \\
\text { e a ausência de feedback das } \\
\text { chefias. }\end{array}$ \\
\hline \multirow{3}{*}{$\begin{array}{c}\text { Estratégias para a } \\
\text { utilização dos } \\
\text { indicadores de } \\
\text { qualidade }\end{array}$} & $\begin{array}{l}\text { Construção e discussão } \\
\text { coletiva/engajamento da } \\
\text { equipe. }\end{array}$ \\
\hline & $\begin{array}{c}\text { Feedback para a equipe de } \\
\text { trabalho. }\end{array}$ \\
\hline & $\begin{array}{c}\text { Trabalhar com a equipe de } \\
\text { maneira participativa. }\end{array}$ \\
\hline
\end{tabular}

Fonte: Elaboração própria (2020).

\section{Dificuldades evidenciadas pelas enfermeiras- líderes para a utilização dos indicadores de qualidade}

Nessa categoria, apresentam-se as dificuldades evidenciadas pelas enfermeiraslíderes para a utilização dos indicadores. A subnotificação é apontada como sendo uma das dificuldades, conforme os depoimentos a seguir.

"Eu acho que a principal dificuldade é a subnotificação" (E5).

"[...] A gente acaba durante o mês não notificando ou não anotando de uma maneira correta; então, muitas vezes, acaba não sendo um valor real para a gente realmente tomar as medidas necessárias ou preventivas" (E4).

"A dificuldade está no profissional que não notifica ou às vezes, não sabe como alimentar as planilhas e não preenche (E12) [...]. Então posso te dizer que é um falso resultado, uma subnotificação" (E12).

"Em relação às subnotificações, eu concordo que são subnotificados; por exemplo, os eventos adversos são subnotificados, registros do perfil epidemiológico e não conformidades" (GFO6).

Os enfermeiros-líderes enfrentam algumas dificuldades no dia a dia perante os indicadores de qualidade, entre elas, a subnotificação de eventos adversos, que, algumas vezes, pode gerar um resultado não fidedigno da realidade do setor ou instituição de saúde e comprometer a tomada de decisão. Vale ressaltar que, ao serem admitidos na instituição do estudo, todos os enfermeiros recebem capacitação acerca dos indicadores e de como devem proceder com seu preenchimento. Sugere-se pensar em capacitações sistemáticas, de modo a garantir o registro das informações.

Percebe-se que os resultados obtidos nas entrevistas são corroborados pelos achados do GF, sendo a subnotificação de eventos adversos uma importante dificuldade na utilização dos indicadores de qualidade. Também se nota nos depoimentos a existência de enfermeiros que não sabem preencher as planilhas dos indicadores, adotadas na instituição e, assim, deixa de alimentá-las, contribuindo para um resultado pouco confiável no final do mês, o que faz os enfermeiros-líderes considerarem um limite importante, para o qual não se pode tomar nenhuma decisão para a mudança nos processos ou ações de prevenção.

Em outros relatos, os enfermeiros consideram a interpretação e aplicabilidade do indicador de qualidade como dificuldades encontradas na operacionalização.

"Acredito que temos dificuldades na interpretação e aplicação dos dados" (E2).

"Hoje acredito que os indicadores da instituição sejam apenas uma estatística, e o objetivo do indicador não pode ser meramente estatístico e sim como uma melhoria" (E3).

"Na prática assistencial está bem difícil de ser utilizado, acredito que as pessoas ainda não 
entenderam e não têm a ideia de pegar o indicador, trabalhar com ele e utilizá-lo na prática. Por exemplo: vamos pegar o indicador de eventos adversos e vamos traçar uma meta para que seja reduzido" (E8).

"Com certeza existe falha na formação, não vimos como notificar e interpretar um indicador, seu significado na assistência, eu nunca tinha visto antes" (GFO4).

Diante desses dados, percebe-se que os enfermeiros têm informações acerca dos indicadores, porém têm dificuldade na interpretação, em reconhecer o que o resultado mensal está apontando sobre a assistência. Notase que os enfermeiros não conseguem ver a aplicabilidade do indicador, que poderia ser utilizado como evidência da prática.

A qualidade das informações, para a utilização dos indicadores, é de suma importância, visto que reflete o resultado do trabalho assistencial, além de permitir que as organizações monitorem seu desempenho e comparem-se com outras instituições, como foi visto em um estudo que desenvolveu indicadores de qualidade para pacientes com patologias agudas ${ }^{(15-16)}$.

As anotações de enfermagem constituem um indicador fundamental para a gerência do cuidado; no entanto são pouco utilizadas pelas instituições de saúde, sendo necessário estabelecer uma estrutura e rotina de coleta e análise dos indicadores, para que os resultados sejam fidedignos à realidade ${ }^{(17)}$. Além disso, o apoio e a participação da alta administração são fundamentais, para melhorar a gestão da qualidade, além de promoverem as mudanças imprescindíveis para o alcance de resultados ${ }^{(18)}$.

Quando se fala de dificuldade na interpretação e na aplicabilidade do indicador, esses resultados convergem com uma pesquisa, também realizada com enfermeiros, na qual eles não conseguiram incorporar todas as fases do gerenciamento dos indicadores, ficando sem saber o que fazer com os números coletados e nem sempre desenvolvendo um planejamento de ações e melhorias no cuidado, a partir dos indicadores $^{(13)}$. Compreende-se por gerenciamento dos indicadores a coleta, a interpretação, análise e o plano de ação. Em relação a essas dificuldades, os enfermeiros podem tê-las por uma lacuna na sua formação sobre o assunto, como foi identificado no presente estudo e vai ao encontro de outra pesquisa, na qual os enfermeiros relataram dificuldades para utilizar os indicadores de processo e a maioria não havia tido nenhum contato teórico ou prático com o tema durante a graduação(8).

Também foi possível constatar a elaboração de planos de ação, a partir dos indicadores, mas não a sua implementação e posterior mensuração.

"Eu acho que, em relação aos planos de ação, precisamos ser mais resolutivos e não só construir um plano de ação, porque a qualidade diz que tem que construir um plano de ação. A gente precisa fazer um plano de ação com vista ao resultado e ver no final do mês se foi efetivo e não só no mês seguinte" (E6).

"Eu acho que a gente ainda não conseguiu colocar em prática as mudanças e como a gente vai aplicar isso (E1) [...] em relação à taxa de infecção: O que estou fazendo para isso? Eu coletei esse dado, eu sei que está ocorrendo e que não está eficiente e eu preciso planejar alguma coisa para que isso aconteça e esse planejamento não está acontecendo. O que estou fazendo com este indicador na prática?" (E1).

Outra dificuldade apontada foi a falta de tempo pela complexidade assistencial do paciente, a rotatividade de colaboradores ou a falta de funcionários, os imprevistos dos plantões, assim como a falta de comprometimento de outros enfermeiros.

"Falta tempo pela questão da complexidade do paciente; eles estão internandos, cada vez mais graves e dependentes e isso exige um tempo mais de assistência. As equipes estão sobrecarregadas $e$ falta profissional qualificado. A rotatividade também é alta, aí falta tempo para os indicadores" (GFO3).

"Falta também muito tempo para fazer os indicadores, ali no meu setor é um setor grande, com pacientes complexos. $O$ quadro de funcionários nunca está completo; então eu tenho que ajudar a equipe. Então, algumas coisas vou fazendo quando dá e durante os plantões" (E11).

"O dia a dia é corrido e nem sempre acontece como prevíamos e daí não conseguimos trabalhar com os indicadores" (GFO6).

"Às vezes nos tornamos tarefeiros, mecanicistas, tu tens que fazer as prescrições, atender às intercorrências, visitar os pacientes [...] e tu acaba se envolvendo na rotina, e esses 
6|Bá o ACP, Amestoy SC, Trindade LL, et al.

processos como dos indicadores acaba ficando para depois" (GF05).

"Falta de tempo e colaboração dos outros colegas enfermeiros" (E6).

A falta de tempo, em razão da complexidade assistencial do paciente e sobrecarga de trabalho, assim como a rotatividade de colaboradores ou a falta de funcionários, há os imprevistos dos plantões e a falta de comprometimento dos colegas. Neste sentido, os enfermeiros e os gestores das instituições precisam repensar o processo de trabalho da enfermagem, analisando criticamente o do enfermeiro, para que consiga atuar no planejamento da assistência, refletindo no desfecho positivo do cuidado ao paciente. Conforme os resultados deste estudo, verifica-se que o maior número de pacientes designados aos profissionais de enfermagem está associado a desfechos negativos, como o aumento da média de permanência e da taxa de infecção urinária ${ }^{(19)}$.

Em relação à rotatividade de pessoal, essa que também é elencada como importante indicador de qualidade da gestão de recursos humanos, especialmente no contexto da enfermagem, merece atenção por parte das lideranças, porque esse fato se alia ao aumento de custos, podendo interferir na qualidade do cuidado e também na manutenção dos níveis de excelência exigidos por programa de qualidade ${ }^{(20)}$.

"A falta de comprometimento dos enfermeiros com os indicadores ainda existe, nós precisamos estar comprometidos com a assistência e, quando olhamos para os indicadores, estamos olhando para a assistência" (E9).

Ainda, diante da utilização dos indicadores de qualidade, os enfermeiros mencionaram a falta de compartilhamento dos dados entre a equipe de enfermagem e multidisciplinar e a ausência de feedback das chefias perante os resultados dos indicadores.

"Aqui no meu setor tem uma pessoa responsável por construir esses indicadores, não são todos os profissionais que participam da construção" (E3).

"No meu setor não tem reunião mensal e quem faz é a enfermeira da tarde, o bom seria que todos participassem, e cada um poderia enxergar o que está dando resultado" (E9).

"Acredito que o maior problema é que sabemos o que precisa ser feito, mas não fazemos, sabemos que precisamos passar para os outros turnos as informações, sabemos que precisamos nos comunicar e não nos comunicamos" (GFO2).

"Não há uma construção coletiva" (GF06).

"Então a chefia fica sabendo do resultado destes indicadores através do preenchimento da planilha e não se senta, não se discute e não se tem esse feedback" (E1).

"Geralmente, em relação ao indicador de infecção, na qual ficamos dez meses sem infecção, quem dava os parabéns e conversava com a equipe era o $\mathrm{CClH}$ [Comissão de Controle de Infecção Hospitalar]" (E10).

"E em relação ao feedback, só acontece quando é algo grave. Em nenhum momento nós do setor discutimos com a coordenação acerca dos indicadores" (E12).

No que se refere à falta de compartilhamento dos indicadores com os demais colegas, os enfermeiros deixam evidente que, na sua maioria, os indicadores estão centralizados em uma pessoa, que detém os dados de uma unidade assistencial. Ainda, relatam a falta de reuniões e a falta de comunicação entre turnos na construção de indicadores, o que gera a fragmentação da informação. Ao falar de feedback, aclaram que só acontece quando é realizado pelo Controle de Infecção Hospitalar ou quando os indicadores sinalizam algo grave.

Cabe ressaltar que a instituição do estudo conta com um enfermeiro em cada turno, um enfermeiro coordenador de área e uma gerente de enfermagem e, na pesquisa, a falta de feedback está relacionada com os enfermeiros dos turnos e as coordenações de áreas. Ao não compartilhar as informações que constam nos indicadores, os enfermeiros perdem a riqueza dos dados, as discussões com a equipe e os detalhes de cada informação contida no indicador, que pode auxiliar na gestão da unidade assistencial e no aprendizado da equipe.

Os enfermeiros-líderes percebem a necessidade da construção e discussão coletiva dos indicadores de qualidade, tanto da equipe de enfermagem como da equipe multidisciplinar. Esse diálogo coletivo auxilia na comunicação entre as áreas e contribui para a consolidação do processo. A assistência em saúde precisa ser pensada e protagonizada por seus atores, buscando melhorias para o processo de trabalho, assim como para o atendimento ao paciente. 
No entanto, mostra-se que o trabalho em equipe constitui um desafio, pelo número e pela diversidade de pessoas e ideias que fazem parte do todo ${ }^{(21)}$. Os resultados da presente pesquisa convergem com o estudo realizado no cenário hospitalar, os participantes mencionam a falta de integração da equipe multiprofissional e relatam a inexistência de coesão entre os profissionais ${ }^{(5)}$. Tal situação revela-se contrária ao preconizado pela PNHOSP de que as equipes multiprofissionais deveriam ser a estrutura nuclear dos serviços de saúde do hospital, formada por profissionais de diferentes áreas e saberes, que irão compartilhar informações e decisões de forma horizontal, estabelecendo-se como referência para os usuários e familiares ${ }^{(10)}$.

No que se refere ao feedback pelas chefias, sua falta pode levar os enfermeiros-líderes se sentirem pouco compromissados com os dados que geram nas unidades mensalmente, assim como pode desestimular a sua utilização, ao mesmo tempo em que é apontado como importante pelos enfermeiros-líderes do estudo.

$O$ envolvimento da equipe de enfermagem e a multidisciplinaridade são essenciais no sentido de reunir todos no processo e comprometê-los pelo resultado assistencial. Em um estudo realizado com enfermeiros, foi visto que a maioria da amostra (54,7\% dos enfermeiros) divulga os dados dos indicadores para a equipe de enfermagem, em momentos de apresentação dos resultados e sua discussão ${ }^{(6)}$. Conforme a PNHOSP, as práticas assistenciais e gerenciais desenvolvidas, a partir da caracterização do perfil dos pacientes, por meio da corresponsabilização das equipes e avaliação de indicadores assistenciais, caracterizam a gestão da clínica(10). Os indicadores podem constituir instrumento para mudar processos internos de trabalho ${ }^{(22)}$ e sua discussão tem assumido um papel estratégico à gestão dos serviços de enfermagem, visto que pode servir para identificar as fragilidades e estabelecer metas objetivando as boas práticas de saúde ${ }^{(23)}$.

\section{Estratégias das enfermeiras-líderes para a utilização dos indicadores de qualidade}

No que diz respeito às estratégias sugeridas pelas enfermeiras-líderessobre a melhor forma de utilização dos indicadores, a construção e discussões coletivas, assim como o engajamento da equipe de enfermagem e a multidisciplinar, surgiram nos depoimentos.
"Acho que a gente poderia, para melhor conduzir a equipe, fazê-la se sentir parte da construção dos indicadores, envolvê-la na construção seria uma estratégia muito boa para que pudesse atuar de uma maneira mais eficaz" (E10).

"Acredito que a equipe multidisciplinar também pode contribuir muito com os indicadores, por exemplo, com a nutrição nós trabalhamos $O$ indicador: dieta prescrita $X$ infundida, acho que isso pode ajudar" (E7).

"Se tu vais desenvolver um plano e vai envolver as pessoas na construção, a equipe, todos vão te auxiliar para a busca daquele objetivo. A gente precisaria ter um momento para poder discutir o processo de trabalho, a assistência" (GFO1).

Observa-se que, apesar de saber como os indicadores de qualidade poderiam ser trabalhados com a equipe de enfermagem e saúde, essas estratégias ainda não são utilizadas na prática em sua totalidade. No caso dos indicadores da equipe de nutrição, já existe uma construção e discussão coletiva com a enfermagem.

Nos depoimentos, os próprios enfermeiros sugeriram uma maneira de como realizar uma discussão com a equipe de enfermagem, o feedback para a equipe de trabalho é essencial para mostrar o resultado e saber em quais aspectos precisa-se melhorar a assistência.

"No mês passado, a gente recebeu um elogio do controle de infecção, por três meses sem infecção e eu trouxe esse resultado para a equipe, eu trouxe esse feedback para eles, porque é importante eles saberem que está dando certo" (E2).

"Os indicadores não é só notificar, precisamos dar um retorno para a equipe. Por exemplo: olha equipe, aqui tivemos tantas infecções, tantas sondas enterais que obstruíram, então vamos juntos traçar um plano para melhorar esse indicador e estabelecer mudanças" (E5).

"Como que tu consegues trabalhar com o técnico: quando tu teve lá no final de mês, cinco perdas de sonda nasoentérica e três foram por obstrução, aí tu consegue trabalhar com a equipe, isso é concreto, daí tu consegue sinalizar para eles" (GF02). 
O enfermeiro-líder, ao utilizar o feedback com a equipe de enfermagem, essa fica sabendo a importância do seu trabalho e no que ele repercute. As falas tanto das entrevistas, como nas sessões do GF, reforçam a ideia de que os indicadores de qualidade precisam ser trabalhados em equipe.

Neste sentido, as instituições de saúde precisam buscar o aprimoramento de seus líderes, habilitando-os a transformarem os dados coletados em informações gerenciais. A liderança auxilia o enfermeiro no alcance dos objetivos da assistência, entre eles, oferecer um cuidado de qualidade.

Mediante esse panorama, a liderança pode ser uma ferramenta que venha a auxiliar o enfermeiro na construção de um ambiente com um clima mais satisfatório aos trabalhadores, por meio do estabelecimento de vínculos profissionais saudáveis e processos dialógicos efetivos entre a equipe de enfermagem e integrantes da equipe multiprofissional ${ }^{(24)}$, ainda contribuindo com as discussões dos indicadores de qualidade.

Outra maneira de trabalhar com os indicadores, que surgiu nos depoimentos das enfermeiras, foi o trabalhar com a equipe de maneira participativa.

"Eu acho que a gente tem que tirar essa ideia de punitivo e partir para o educativo. Uma coisa bem legal que eu tenho visto, por exemplo, quando um funcionário aplica uma medicação errada, um tempo atrás tínhamos que advertir, só que temos que pensar que não foi o funcionário, tem uma cascata de coisas por trás do erro, nós temos que parar com essa coisa de punir as pessoas pelos erros e sermos educativos" (GFO3).

"Todas as coisas que a gente estuda, nós estudamos juntos, de acordo com as demandas. A gente precisa envolver a equipe no processo, para eles se sentirem importantes" (E5).

Envolver a equipe no desempenho, permitir a participação no processo, reconhecer o trabalho de todos com sistema de recompensa e avaliação de desempenho, foram algumas práticas gerenciais desenvolvidas em uma instituição, para alcançar a acreditação por excelência. Com mais interação, em todas as etapas do processo de acreditação, os funcionários passaram a ter uma visão do macro, dentro do seu setor e da própria instituição ${ }^{(18)}$. No hospital do estudo, ainda não existe avaliação de desempenho dos trabalhadores, prática que pode ser pensada e correlacionada com os indicadores assistenciais, a ser realizada de maneira periódica e sistematizada.

A educação permanente pode favorecer a disseminação das informações acerca de instrumentos de melhoria da qualidade, a fim de estimular a adoção de práticas mais efetivas e eficazes, além de oferecer respaldo às atividades desenvolvidas ${ }^{(12)}$, levando em conta que os temas, para a realização da educação permanente, emergem do próprio processo de trabalho da equipe. Cabe mencionar que a educação permanente é importante por permitir atualização das práticas realizadas cotidianamente pelos profissionais e construção de relações e processos que vão das equipes em atuação às práticas institucionais, pois sinaliza-se que, durante a formação em enfermagem, os aspectos técnicos, muitas vezes, são mais valorizados em detrimento dos aspectos gerenciais, os quais são também necessários na prática assistencial, em que necessitam tomar decisões que vão impactar na qualidade e no resultado institucional ${ }^{(5)}$.

Na perspectiva da PNHOSP, destaca-se a preocupação quanto à formação, ao desenvolvimento e à gestão da força de trabalho; desse modo, os espaços de produção das ações e dos serviços de saúde no SUS devem constituir-se em campo de prática para o ensino, a pesquisa e a incorporação tecnológica em saúde, cabendo aos hospitais integrantes do SUS desempenhar um importante papel na formação. A educação permanente e a participação em ações de formação de novos profissionais de saúde também devem integrar programas e políticas de formação em saúde ${ }^{(10)}$. Identifica-se um movimento ainda muito incipiente por parte dos hospitais, em realizar programas voltados para a formação em saúde, de modo que, em alguns momentos, encontram-se profissionais pouco capacitados para o atendimento aos pacientes do sistema de saúde.

Trabalhar de maneira educativa pode ser mais fácil para a equipe entender a importância dos indicadores de qualidade e foi elencado pelos enfermeiros-líderes como estratégia para trabalhar com a equipe essa temática. A articulação entre a assistência, gerência e a educação é necessária e imprescindível, no que tange à acreditação hospitalar e à melhoria da qualidade do cuidado $^{(21)}$. Conforme a PNHOSP, o programa de educação permanente em saúde deve ser oferecido aos profissionais de saúde das equipes dos hospitais, baseado no aprendizado 
em serviço, no qual o aprender e ensinar se incorporam ao cotidiano dos hospitais(10).

Quando a equipe está envolvida no monitoramento, acompanhamento e na análise dos indicadores, além de conhecer os resultados, ela é descentralizada, produzindo mais autonomia no trabalho da equipe e dos líderes ${ }^{(18)}$. As enfermeiras sabem o que precisam fazer para aproximar a equipe de enfermagem e saúde dos indicadores de qualidade, porém ainda não conseguiram efetivar essa prática em sua totalidade. Assim, sugere-se que a instituição possa proporcionar momentos de interação da equipe de enfermagem e saúde, contribuindo com a sua aproximação e favorecendo a comunicação às equipes.

Vale lembrar que, ao trabalhar com a gestão da qualidade, precisa-se investir na gestão de pessoas; as organizações devem focar efetivamente no desenvolvimento e na valorização dos trabalhadores. Sendo assim, a comunicação e a adoção de reuniões de repasse são estratégias gerenciais para alcançar níveis de excelência. Neste estudo, as reuniões acontecem semanalmente, entre alta administração e gerentes; da mesma maneira, os gerentes repassam o que foi discutido para a sua equipe, o que se mostrou fundamental para envolver toda a equipe e alcançar metas ${ }^{(18)}$. Estudo pondera, ainda, que alinhar os indicadores propostos na gestão estratégica da instituição é uma forma de contribuir para o despertar da busca de conhecimento gerencial e a aproximação aos conceitos teóricos administrativos com a prática da enfermagem ${ }^{(4)}$.

Também, emerge a necessidade de reforçar a translação do conhecimento entre o ensino e os serviços de saúde, concretizando um processo de formação que reconheça as modificações ocorridas, diante dos novos modelos assistenciais e gerenciais, o que muda o processo de trabalho em saúde e exige mudança no perfil profissional, requerendo uma formação com aptidões para a tomada de decisão, comunicação, liderança, o gerenciamento e a educação(21). Salienta-se que cabe aos centros formadores a responsabilidade de capacitar o futuro profissional ao desenvolvimento de tais saberes, repensando suas estratégias e prioridades de ensino, a fim de atenderem às necessidades de saúde com efetividade ${ }^{(25)}$.

\section{CONCLUSÃO}

Neste estudo, os enfermeiros identificaram as dificuldades evidenciadas, para a utilização dos indicadores de qualidade, assim como propõem estratégias para a utilização dos indicadores de qualidade no âmbito hospitalar.

Os líderes percebem que a subnotificação de eventos adversos e o não preenchimento da planilha dos indicadores podem contribuir com resultados não fidedignos da assistência, ao mesmo tempo, a interpretação e aplicabilidade do indicador de qualidade são também dificuldades encontradas na operacionalização. Como sugestão, a instituição poderá pensar em reestruturar seus formulários e o processo de notificação, de maneira a instigar as notificações pelos enfermeiros e demais profissionais; ao mesmo tempo, fazer busca ativa dos incidentes, por auditorias internas, o que poderá auxiliar nos resultados de gestão. A falta de tempo, em decorrência da complexidade assistencial do paciente, a rotatividade de colaboradores ou o número reduzido de funcionários, as intercorrências diárias, assim como o não comprometimento de alguns enfermeiros também foram relatadas pelos participantes, além da falta de compartilhamento dos dados e feedback por parte das coordenações de áreas.

No que se refere às estratégias dos enfermeiros, para a utilização dos indicadores de qualidade, eles citaram a construção e a discussão coletiva, assim como o engajamento da equipe de enfermagem e a multidisciplinar, ainda que não utilizadas na prática em sua totalidade. Neste sentido, a instituição poderá pensar em maneiras de reunir os atores envolvidos, em busca de melhores resultados e soluções. Assim sendo, o feedback para a equipe e o trabalho educativo são essenciais para mostrar o resultado e saber do que se precisa para melhorar a assistência.

Em relação às limitações do estudo, destaca-se o receio dos participantes em manifestarem suas percepções sobre os indicadores de qualidade, dificuldades e estratégias quanto à sua utilização no âmbito hospitalar, visto que são funcionários da instituição. No entanto a estratégia de coleta de dados com entrevistas e o grupo focal foram fundamentais para conhecer as percepções individuais e do grupo de enfermeiros-líderes.

Em relação a futuros estudos, sugere-se pensar em novas estratégias de técnica de coleta de dados, como o preenchimento de 
10|Bá o A C P, Amestoy SC, Trindade LL, et a I.

questionários com entrega codificada e com outros profissionais além dos enfermeiros.

Os resultados do estudo implicam de forma positiva na prática assistencial dos enfermeiros, tendo em vista que reforçam a necessidade de qualificar o gerenciamento do cuidado, por meio da utilização dos indicadores de qualidade, reconhecendo as dificuldades e estratégias para a sua implementação no ambiente hospitalar. Para tanto, é necessário que as instituições de saúde também se sensibilizem, em instrumentalizar os enfermeiros-líderes, de maneira a capacitar seus gestores para a melhor utilização dos indicadores de qualidade, visto que as instituições de ensino não preenchem todas as necessidades requeridas pelo mercado do trabalho, ao mesmo tempo, em que as instituições de ensino devem repensar no conteúdo ministrado acerca dos indicadores de qualidade, dando subsídios para os futuros enfermeiros.

Notou-se que o grupo de enfermeiras do estudo ainda enfrenta dificuldades no que se refere à proatividade, não conseguindo propor solução aos problemas apontados pelos indicadores. Da mesma forma, é necessário que os hospitais se voltem para as políticas de saúde, em especial, à PNHOSP, no sentido de qualificar a atenção hospital e a gestão da clínica.

\section{REFERÊNCIAS}

1 - Kristensen S, Hammer A, Bartels A, Suñol R, Groene O, Thompson CA, et al. Quality management and perceptions of teamwork and safety climate in european hospitals. Int J Qual Health Care 2015;27(6): 499-506. DOI: 10.1093/intqhc/mzv079

2 - Santos RS, Lima FM, Hora JC, Leão DBM. Indicadores de qualidade aplicados na assistência de enfermagem em cuidados paliativos: revisão integrative de literature. Enferm Foco 2020 [citado em 8 jun 2020]; 11(2):191-7. Available in: https://api-wordpress.paliativo.org.br/wpcontent/uploads/2020/12/ARTIGO 2652-211161-PB.pdf

3 - Foebel AD, Van Hout HP, Van der Roest HG, Topinkova E, Garms-Homolova V, Frijters D, et al. Quality of care in European home care programs using the second generation interRAl Home Care Quality Indicators (HCQIs). BMC Geriatr. 2015;14(15):148. DOI: 10.1186/s12877-015-0146$\underline{5}$
4 - Fugaça NPA, Cubas MR, Carvalho DR. Use of balanced indicators as a management tool in nursing. Rev Latino-Am Enfermagem 2015;23(6):1049-56. DOI: 10.1590/0104$\underline{1169.0670 .2648}$

5 - Braga AT, Pena MM, Melleiro MM. Métrica de indicadores assistenciais de hospitais certificados. Rev Enferm UFPE 2018;12(3):665-75. DOI: 10.5205/1981-8963-v12i3a230715p665-675-2018

6 - Cavalcante PS, Rossaneis MA, Haddad MCL, Gabriel CS. Indicadores de qualidade utilizados no gerenciamento da assistência de enfermagem hospitalar. Rev Enferm UERJ 2015;23(6):787-93. DOI: $10.12957 /$ reuerj.2015.7052

7 - Silva VLS, Camelo SHH, Soares MI, Resck ZMR, Chaves LDP, Santos FC, et al. Leadership practices in hospital nursing: a self of manager nurses. Rev Esc Enferm USP 2016;51:e03206. DOI: 10.1590/S1980-220X2016024403206

8 - Menezes PIFB, D'Innocenzo M. Dificuldades vivenciadas pelo enfermeiro na utilização de indicadores de processos. Rev Bras Enferm. 2013;66(4):571-7. DOI: 10.1590/s003471672013000400016

9-Tang $X$, Chen X, Pang $Y$, Zhou L. The development of quality indicators for home care in China. Int J Qual Health Care 2018;30(3):20818. DOI: $10.1093 /$ intahc/mzx202

10 - Brasil. Ministério da Saúde. Portaria 3.390, de 30 de dezembro de 2013. Política Nacional de Atenção Hospitalar (PNHOSP) no âmbito do Sistema Único de Saúde (SUS), estabelecendo-se as diretrizes para a organização do componente hospitalar da Rede de Atenção à Saúde (RAS). Brasília, DF: MS; 2013.

11 - Rossaneis MA, Gabriel CS, Haddad MCFL, Melo MRAC, Bernardes A. Indicadores de qualidade utilizados nos serviços de enfermagem de hospitais de ensino. Rev Eletrônica Enferm. 2014;16(4):769-76. DOI: 10.5216/ree.v16i4.22956

12 - Marreiro LAA, Ribeiro RAA, Santos SR, Albuquerque GE, Carvalho GDA. Perceptions and experiences of nurses managers concerning quality indicators. Rev Enferm UFPE 2015;9(4):7255-63. DOI: 10.5205/1981-8963v9i4a13581p7255-7263-2015 
13 - Silveira TVL, Prado Júnior PP, Siman AG, Amaro MOF. The importance of using quality indicators in nursing care. Rev Gaúcha Enferm. 2015;36(2):82-8. DOI: $\quad$ 10.1590/19831447.2015.02.47702

14 - Minayo MCS. O desafio do conhecimento: pesquisa qualitativa em saúde. 14a ed. São Paulo: Hucitec; 2014.

15 - Morris JN, Berg K, Topinkova E, Gray LC, Schachter E. Developing quality indicators for inpatient post-acute care. BMC Geriatr. 2018;18(1):161. DOI: 10.1186 / s12877-018-0842$\underline{z}$

16 - Bao H, Yang F, Wang X, Su S, Liu D, Fu R, et al. Developing a set of quality indicators for breast cancer care in China. Int J Qual Health Care 2015;27(4):291-6. DOI: 10.1093/intqhc/mzv042

17 - Rossaneis MA, Gabriel CS, Haddad MCFL, Melo MRAC, Bernardes A. Indicadores de qualidade da assistência: opinião de enfermeiros gerentes de hospitais de ensino. Cogitare Enferm. 2015;20(4):798-804. DOI: 10.5380/ce.v20i4.41734

18 - Siman AG, Cunha SGS, Martins ES, Brito MJM. Estratégia do trabalho gerencial para alcance da acreditação hospitalar. Rev Min Enferm. 2015;19(4):815-22. DOI: 10.5935/1415$\underline{2762.20150063}$

19 - Magalhães AMM, Costa DG, Riboldi CO, Mergen T, Barbosa AS, Moura GMSS. Association between workload of the nursing staff and patient safety outcomes. Rev Esc Enferm USP 2017;51:e03255. DOI: 10.1590/s1980$\underline{220 \times 2016021203255}$

20 - Oliveira JLC, Matsuda LM. Vantagens e dificuldades da acreditação hospitalar: a voz dos gestores da qualidade. Esc Anna Nery 2016;20(1):63-9. DOI: 10.5935/14148145.20160009

21 - Oliveira JLC, Hayakawa LY, Versa GLGS, Padilha EF, Marcon SS, Matsuda LM. atuação do enfermeiro no processo de acreditação: percepções da equipe multiprofissional hospitalar. Rev Baiana Enferm. 2017; 31(2):e17394. DOI: 10.18471/rbe.v31i2.17394

22 - Amaral JAB, Spiri WC, Bocchi SCM. indicadores de qualidade em enfermagem com ênfase no centro cirúrgico: revisão integrativa da literatura. Rev SOBECC 2017;22(1):42-51. DOI: 10.5327/Z1414-4425201700010008

23 - Báo ACP, Amestoy SC, Moura GMSS, Trindade LL. Quality indicators: tools for the management of best practices in Health. Rev Bras Enferm. 2019;72(2):360-6. DOI: 10.1590/00347167-2018-0479

24 - Garcia BL, Thofehrn MB, Porto AR, Moura PMM, Carvalho LA, Fernandes HN. Relação entre liderança e vínculos profissionais: percepção de enfermeiros. Rev Pesq Saúde 2017 [citado em 8 jun 2020]; 18(2):114-8. Available in: http://www.periodicoseletronicos.ufma.br/index. php/revistahuufma/article/view/6715

25 - Soares MI, Camelo SHH, Resck ZMR, Terra FS. Saberes gerenciais do enfermeiro no contexto hospitalar. Rev Bras Enferm. 2016;69(4):676-83. DOI: 10.1590/0034-7167.2016690409i

Nota: Este artigo é um recorte da dissertação: "Indicadores de qualidade: ferramentas do enfermeirolíder para o gerenciamento do cuidado", como requisito parcial para a obtenção de título de mestre da Faculdade de Enfermagem da Universidade Federal de Pelotas/RS, 2016.

Recebido em: $10 / 08 / 2020$

Aprovado em: 22/02/2021 